\title{
Severe Defects in the Macrophage Barrier to Gut Microflora in Inflammatory Bowel Disease and Colon Cancer
}

\author{
CARLOS A. RUBIO ${ }^{1}$ and PETER T. SCHMIDT ${ }^{2}$ \\ ${ }^{1}$ Gastrointestinal and Liver Research Laboratory, Department of Pathology, \\ and ${ }^{2}$ Department of Medicine Solna, Center for Digestive Diseases, \\ Karolinska Institute and University Hospital, Stockholm, Sweden
}

\begin{abstract}
The continuity of the subepithelial band of lamina propria-indigenous macrophages (SBLP-M) discourages commensal gut bacteria from invading the host. In this Review we analyzed the impact of a disintegrating SBLP-M in inflammatory bowel disease (IBD), which results in microbiota inflow, inadequate immune responses and IBD-associated colon cancer. In previous work, we analyzed endoscopic biopsies taken from normal-looking descending colon in 247 patients with IBD, and 167 from control patients without IBD. Sections immunostained for cluster of differentiation 68 (CD68) protein showed no inflammatory changes. In IBD, the band of CD68+ $S B L P-M$ was fragmented or minute in 59\% (47/80) and absent in 9\% (7/80). In contrast, only $31 \%$ (51/167) of the biopsies from control patients had a fragmented/minute band of CD68+SBLP$M$ and this band was not absent in any $(p<0.05)$. The finding that the band of $C D 68+S B L P-M$ was fragmented to totally lost in IBD suggests a long-lasting defect in the barrier against the gut microbiome in IBD. The lack of ongoing inflammation in colonic biopsies should rule-out the participation of bone marrow-derived inflammation-elicited macrophages in loss of the barrier. Today, it is widely accepted that dysbiosis and inappropriate immune response to microbial flora play a pivotal role in the pathogenesis and development of IBD-associated colon cancer. Based on present knowledge, it is submitted that defects in the SBLP-M barrier in IBD encourage the trespassing
\end{abstract}

This article is freely accessible online.

Correspondence to: Carlos A. Rubio, MD, PhD, Gastrointestinal and Liver Pathology Research Laboratory, Department of Pathology, Karolinska Institute and University Hospital, 17176, Stockholm, Sweden. Tel: +46 851774527, Fax: +46 851774524, e-mail: Carlos.Rubio@ki.se

Key Words: Colon, macrophages, gut-microflora, CD68, ulcerative colitis, Crohn's colitis, review. of the gut microflora into the host, thereby destabilizing host immunity. These events in concert may play the ultimate pivotal role in the evolution of colon cancer in patients with IBD.

In recent years, much concern has been raised over the role played by the gut microbiome and gut inflammation, and their effect on the host immune system and colonic carcinogenesis. The cross-talk between the gut commensal microbiota and the subepithelial resident (indigenous) macrophages encourages the creation of a protective barrier of macrophages. Under normal conditions, an unbroken barrier of colonic macrophages is essential in hampering host invasion by commensal bacteria. The mechanisms whereby the gut bacteriome reaches the host remains unclear. In this review, we discuss the possible impact of a defective macrophage barrier in the gut on the putative trespassing of the gut bacteriome in inflammatory bowel disease (IBD), their effect on the host immune system and the development of IBD-associated colonic carcinoma.

\section{Macrophages in the Colonic Mucosa}

The mononuclear phagocytic system is composed of blood leucocytes (monocytes and macrophage), tissue macrophages (histiocytes), and Kupffer cells. In this superfamily, two macrophage systems are recognized, one derived from bonemarrow lineage-committed monocytes (BMMs), the other of resident indigenous macrophages $(1,2)$. BMMs circulate in the blood, but when chemo-attractants are released from tissues injured by trauma or by infections, BMMs leave the circulation. In the chemically different hostile microenvironment, BMMs differentiate into macrophages. Of the resident indigenous macrophages, the gut macrophages comprise the largest macrophage pool in the body (3). In the non-inflamed mucosa, gut macrophages are inflammation anergic, but retain scavenger and host defense function, a perfect attribute for macrophages in close proximity to gut microbiota. 


\section{Submucosal Macrophages in IBD}

Under normal conditions, indigenous gut macrophages gather as a continuous band in the subepithelial lamina propria (SBLP) of the colon (4). SBLP macrophages are crucial in deterring commensal gut microbiota from attacking their host by rapidly eliminating gut microflora (5-7). By producing over 100 secretory products, gut SBLP macrophages choreograph pro-inflammatory and host defense activities $(8,9)$. Recognition receptors allow SBLP macrophages to detect, engulf and kill a complex and vast number of different bacteria, bacteriophages, fungi, and viruses (gut virome), collectively called gut microbiota (10, 11). During an inflammatory response, both resident gut SBLP macrophages and inflammation-elicited macrophages, derived from blood-circulating monocytes, play crucial roles in the control of infection by actively pursue invading microorganisms, through degradation and release of inflammatory mediators (7).

\section{Breakdown of the SBLP Macrophages in IBD}

A breakdown of the integrity of SBLP macrophages in IBD allow both alien and commensal gut bacteria to trespass that barrier, resulting in host invasion, thereby altering natural host immunity. In previous work, we analyzed the characteristics of the band of CD68+ SBLP macrophages in colonic biopsies without ongoing inflammation (to exclude the participation of inflammation-elicited macrophages) in patients with protracted IBD (12). Left colon biopsies without ongoing inflammation harvested from 247 patients with endoscopically normal left colon were reviewed. Eighty were from patients with IBD ( 27 had ulcerative colitis in remission and 53 had right-sided Crohn's colitis. The remaining 167 were non-IBD control patients (90 had chronic colonic diarrhea, 63 were enrolled in a surveillance colorectal cancer (CRC) program, seven had microscopic colitis (four collagenous and three lymphocytic), and seven had miscellaneous colonic conditions (two infectious colitis, two HIV-associated colitis, one Bechterew colitis and two melanosis coli). Sections were stained with hematoxylin and eosin and immunostained for cluster of differentiation 68 protein (CD68). CD68 is a glycosylated lysosomal membrane protein expressed in the cytoplasmic granules of cells with phagocytic properties. The pattern of CD68+ macrophages found in the SBLP were divided into four types: i) Continuous, when an unbroken band of CD68+ macrophages was found for the entire section; ii) fragmented, when one or more segments of that band were missing from the entire section; iii) minute, when only five or more CD68+ macrophages were found in the entire section; and iv) absent when $\leq 4$ consecutive CD68+ macrophages were found in the entire section. In IBD, the band of SBLP CD68+ macrophages was fragmented or minute in 59\% (47/80) and negative in 9\% (7/80). In contrast, only $31 \%(51 / 167)$ of the control patients had a fragmented/minute band, and a band was absent from none. The difference in the continuity of the CD68+ macrophage band between IBD and controls was significant $(p<0.05)$.

The cross-talk between the gut commensal microbiota and the gut CD68+ SBLP macrophages results in the formation of a barrier of SBLP macrophages (12). This band is the first line of defense (gatekeeper) against gut-microbiota intrusion deep into the LP $(4,12)$. Before reaching the LP, the microbiota must successively overcome several obstacles: i) The thick mucous layer covering the epithelial surface (13); ii) the natural tight junctions that intertwine colonic epithelial cells (14); iii) the natural peptide family of trefoil factors 1 , 2 , and 3 (15); and the innate antimicrobial enzyme lysozyme (16-18). Thus, a breach of the natural continuous SBLP macrophage barrier only arises after the other aforementioned barriers have been overcome. When all these barriers have successively been penetrated, dendritic cells (19) and remnant deeper LP macrophages would be responsible for counteracting invading microbiota. Hence, the proportion of cases with a fragmented/minute or even lacking a CD68+ SBLP macrophage band was significantly higher in patients with IBD than in controls, suggesting a long-lasting lack in continuity of the CD68+ SBLP macrophage barrier against the altered IBD-associated gut microbiome. The lack of ongoing inflammation in colonic biopsies ruled-out the participation of bone marrow-derived inflammation-elicited macrophages in the loss of the SBLP macrophage band.

\section{The Normal Colonic Bacterial Flora}

Healthy gut microbiomes as assessed by sequencing are consistently dominated by bacteria of two phyla, Bacteroidetes and Firmicutes (20). A core set of more than 50 distinct bacterial genera is found, among them, Enterobacteria, Enterococci, Lactobacilli, Clostridia, Bacteroides, gram-positive non-sporing anaerobes (Eubacteria and Bifidobacteria) (21). Over 1,000 bacterial species of the gut have now been characterized, providing a significant inventory of bacterial constituents. Interestingly, molecular phylogenetics has led to the reclassification of many of these species in the past 20 years (20). Of particular interest, species within Bacteroides, previously considered the most prevalent and abundant bacterial genus in the gut, have been reclassified into five genera: Alistipes, Prevotella, Paraprevotella, Parabacteroides, and Odoribacter (22).

Compiled data from these studies (22) identified 2,172 species isolated from human beings, classified into 12 different phyla, of which $93.5 \%$ belonged to Proteobacteria, Firmicutes, Actinobacteria and Bacteroidetes. Three of the 12 identified phyla included only one species isolated from humans, namely 
an intestinal species, Akkermansia muciniphila, the only known representative of the Verrucomicrobia phylum. In humans, 386 of the identified species are strictly anaerobic and will therefore generally be found in mucosal regions such as the oral cavity and the gastrointestinal tract (22).

\section{Altered Bacteriome in Sporadic Colonic Cancer}

Tilg et al. recently reviewed the important role of Fusobacterium nucleatum, Escherichia coli, and Bacteroides fragilis in sporadic colon cancer (23). The intestinal microbiota also comprises viruses and fungi, but the role of viruses and fungi in CRC has not been investigated. Rubinstein et al. demonstrated that $F$. nucleatum, through Fusobacterium adhesin $\mathrm{A}(\operatorname{FadA})$, which binds to E-cadherin, thereby activating the $\beta$-catenin signaling pathway, resulting in induction of oncogenic and inflammatory responses (24). Importantly, FadA gene expression in human CRC tissue was found to be extraordinarily high compared with healthy controls, F. nucleatum increased tumor multiplicity in the adenomatous polyposis coli/multiple intestinal neoplasia (ApcMin/+) model of intestinal tumorigenesis (25). E. coli is a gut commensal, although certain strains have acquired the ability to promote intestinal inflammation and to produce toxins such as colibactin with oncogenic potential (26). Colonization of a colon cancer-associated $E$. coli strain in ApcMin/+ mice resulted in a marked increase in the number of polyps, suggesting that certain E. coli strains might indeed promote tumorigenesis. Similarly to E. coli, largely experimental evidence supports a role for $B$. fragilis in intestinal tumorigenesis. B. fragilis comprises about $1-2 \%$ of the commensal microbiota in most humans.

It should be mentioned that the colonic microbiota also includes viruses and fungi. Nonetheless, the role of viruses and fungi in colon carcinogenesis remains unclear (27).

\section{Altered Bacteriome in IBD}

An altered gut microbiota has repeatedly been found in patients with IBD (28-34). The concept of an altered gut microbiota, called dysbiosis (epitomized by the expansion of bacterial taxa), is possibly the most significant development in IBD research in the past decade. In recent years, the types of bacteria found in IBD have intensively been investigated. Lower levels of B. bifidobacterium, Faecali bacterium, Saccharomyces cerevisiae and Lactobacillus are found but increased proportions of Basidiomycota/Ascomycota, and levels of Proteobacteria, Fusobacterium spp, E. faecalis, Neisseriaceae blauti, Candida albicans, Haemophilus influenzae and other Haemophilus species, and aggressive adherent invasive $E$. coli pathovar have been found (28-34). In addition, Kostic et al. reported a decrease in alpha diversity, Clostridia, Ruminococcaceae erysipelotrichales,
Bacteroidales and Clostridiale in IBD (29), whereas Gevers et al. identified a strong correlation of Crohn's disease with increased abundance of Enterobacteriaceae, Pasteurellacaea, Veillonellaceae, and Fusobacteriaceae, and a decreased abundance of $E$. coli was also found within intestinal macrophages (34).

\section{Altered Bacteriome in IBD-associated Colon Cancer}

The defects in the SBLP macrophage barrier in IBD would allow trespassing of the indigenous and of the alien microflora into the host, an anomaly that alters natural host immunity. Today, it is widely accepted that dysbiosis and inappropriate immune response to microbial flora play a pivotal role in the pathogenesis and development of IBD-associated CRC (3539). These findings have been substantiated in experimental animals $(23,40-42)$. A study by $\mathrm{Wu}$ et al. revealed that enterotoxigenic B. bifidobacterium, which secretes the toxin BFT, induces colitis and colonic tumors in ApcMin/+ mice (43). Detection of certain bacterial strains, namely the combination of F. nucleatum, Bacteroides clarus, Roseburia intestinalis, and Clostridium hathewayi, demonstrated a sensitivity $>90 \%$ and a specificity $>80 \%$ for CRC (44). Colonic polyposis in $\mathrm{Apc}^{\mathrm{Min} /+}$ mice is accompanied by accumulation of microbes in polyps, triggering local inflammatory responses. Native Americans with a low CRC risk exhibit lower stool levels of total short-chain fatty acid butyrate compared with African/Caucasian Americans having a considerably higher CRC risk (45). It is likely that a combination of alterations, rather than increased or decreased abundance of a particular strain in the intestinal microbiota promotes tumorigenesis. Remarkably, patients with Crohn's disease exhibit similar microbial alterations (e.g., increased abundance of E. coli and F. nucleatum) as seen in CRC (46), suggesting not only inflammation but also a shared dysbiosis may contribute to IBD-associated CRC (23).

\section{Host Immunity and Colon Cancer}

There is growing evidence that the host immune system critically controls intestinal carcinogensis (28, 47). Accumulating evidence indicates that intestinal microflora has protective, metabolic, trophic and immunological functions and are able to establish cross-talk with the immune component of mucosal immunity, comprising cellular and soluble elements. Microbiota communicate with the immune system through tolllike receptor signaling (48) and inflammasome sensing nucleotide-binding oligomerization domain (NOD)-like receptors (49) have been implicated in the progression of colonic tumorigenesis. NOD1 recognizes bacterial antigens, which triggers an immune response, and NOD1 deficiency resulted in increased development of tumors in $\mathrm{Apc}^{\mathrm{Min} /+}$ mice and in an inflammation-related tumor model (50). 


\section{Conclusion}

Today, it is widely accepted that dysbiosis and inappropriate immune response to microbial flora play a pivotal role in the pathogenesis and development of IBD-associated colon cancer. Based on present knowledge, it is submitted that defects in the SBLP-macrophage barrier in IBD encourages the trespassing of the gut microflora into the host, thereby destabilizing host immunity. These events in concert play a pivotal role in the evolution of colon cancer in patients with IBD.

\section{References}

1 Cassol E and Poli G: Macrophage polarization in health and disease.Sci World J 11: 2391-2402, 2011.

2 Smith PD, Smythies LE, Shen R, Greenwell-Wild T, Gliozzi M and Wahl SM: Intestinal macrophages and response to microbial encroachment. Mucosal Immunol 4: 31-42, 2011.

3 Nakanishi Y, Sato T and Ohteki T: Commensal Gram-positive bacteria initiates colitis by inducing monocyte/macrophage mobilization. Mucosal Immunol 8: 152-160, 2015.

4 Rubio CA: Macrophage-associated chronic diarrhea of unknown origin. Preliminary observations. In Vivo 19: 927-929, 2005.

5 Kamada N, Hisamatsu T, Okamoto S, Sato T, Matsuoka K, Arai K, Nakai T, Hasegawa A, Inoue N, Watanabe N, Akagawa KS, and Hibi T: Abnormally differentiated subsets of intestinal macrophage play a key role in Th1-dominant chronic colitis through excess production of IL-12 and IL-23 in response to bacteria. J Immunol 175: 6900-6908, 2005.

6 Peiser L and Gordon S: The function of scavenger receptors expressed by macrophages and their role in the regulation of inflammation. Microbes Infect 3: 149-159, 2001.

7 Grainger JR, Konkel JE, Zangerle-Murray T and Shaw T: Macrophages in gastrointestinal homeostasis and inflammation. Pflugers Arch 469: 527-539, 2017.

8 Kühl AA, Erben U, Kredel LI and Siegmund B: Diversity of intestinal Macrophages in inflammatory Bowel Diseases. Front Immunol 6: 613-616, 2015.

9 Liddiard K, Rosas M, Davies LC, Jones SA and Taylor PR: Macrophage heterogeneity and acute inflammation. Eur $\mathrm{J}$ Immunol 41: 2503-2508, 2011.

10 Hedin C. R, McCarthy NE, Louis P, Farguharson F, McCartney S, Taylor $\mathrm{K}$ and Lindsay J: Altered intestinal microbiota and blood T-cell phenotype are shared by patients with Crohn's disease and their unaffected siblings. Gut 63: 1578-1586, 2014.

11 Zhou M, He J, Shen Y, Zhang C, Wang J and Chen Y: New Frontiers in genetics, gut microbiota, and immunity: A Rosetta Stone for the pathogenesis of inflammatory bowel disease. Biomed Res Int 17: 8201672, 2017.

12 Rubio CA, Langner C and Schmidt PT: Partial to complete abrogation of the subepithelial macrophage barrier against the gut microbiota in patients with ulcerative colitis and Crohn's colitis. Histopathology 72: 580-587, 2018.

13 Johansson M, Phillipson M, Petersson J, Velcich A, Holm L and Hansson G: The inner of the two MUC2 mucin-dependent mucous layers in colon is devoid of bacteria. Proc Natl Acad Sci USA 105: 15064-15069, 2008.
14 Landy J, Ronde E, English N, Clark SK, Hart AL, Knight SC, Ciclitira PJ and Al-Hassi HO: Tight junctions in inflammatory bowel diseases and inflammatory bowel disease associated colorectal cancer. World J Gastroenterol 22: 3117-3126, 2016.

15 Srivastava S, Kedia S, Kumar S, Pratap Mouli V, Dhingra R, Sachdev V, Tiwari V, Kurrey L, Pradhan R and Ahuja V: Serum human trefoil factor 3 is a biomarker for mucosal healing in ulcerative colitis patients with minimal disease activity. J Crohns Colitis 9: 575-579, 2015.

16 Fahgren A, Hammarström S, Danielsson $\AA$ and Hammarström ML: Increased expression of antimicrobial peptides and lysozyme in colonic epithelial cells of patients with ulcerative colitis. Clin Exp Immunol 131: 90-101, 2003.

17 Vaishnava S, Yamamoto M, Severson KM, Ruhn KA, Yu X, Koren O, Ley R, Wakeland EK and Hooper LV: The antibacterial lectin Reg III gamma promotes the spatial segregation of microbiota and host in the intestine. Science 334: 255-258, 2011.

18 Rubio CA: Increased production of lysozyme associated with bacterial proliferation in Barrett's esophagitis, chronic gastritis, gluten-induced atrophic duodenitis (celiac disease), lymphocytic colitis, collagenous colitis, ulcerative colitis and Crohn's colitis. Anticancer Res 35: 6365-6372, 2015.

19 Magnusson MK, Brynjólfsson SF, Dige A, Uronen-Hansson H, Börjesson LG, Bengtsson JL, Gudjonsson S, Öhman L, Agnholt J, Sjövall H, Agace WW and Wick MJ: Macrophage and dendritic cell subsets in IBD: ALDH+ cells are reduced in colon tissue of patients with ulcerative colitis regardless of inflammation. Mucosal Immunol 9: 171-182, 2016.

20 Lloyd-Price, Abu-Ali G and Huttenhover C: The healthy human microbiome. Genome Med 8: 51-56, 2016.

21 Hill MJ and Drasar BS: The normal colonic bacteria flora. Gut 16: 318-23, 1975.

22 Thursby $\mathrm{E}$ and Juge $\mathrm{N}$ : Introduction to the human gut microbiota. Biochem J 474: 1823-1836, 2017.

23 Tilg H, Adolph TE, Gerner RR and Moschen AR: The intestinal microbiota in colorectal cancer. Cancer Cell pii: S1535-6108, 2018. https://doi.org/10.1016/j.ccell.2018.03.004

24 Rubinstein MR, Wang X, Liu W, Hao Y, Cai G and Han YW: Fusobacterium nucleatum promotes colorectal carcinogenesis by modulating E-cadherin/beta-catenin signaling via its FadA adhesin. Cell Host Microbe 14: 195-206, 2013.

25 Kostic A, Chun E, Robertson L, Glickman JN, Gallini CA, Michaud M, Clancy TE, Chung DC, Lochhead P and Hold GL: Fusobacterium nucleatum potentiates intestinal tumorigenesis and modulates the tumor-immune microenvironment. Cell Host Microbe 14: 207-215, 2013.

26 Denizot J, Desrichard A, Agus A, Uhrhammer N, Dreux N, Vouret-Craviari V, Hofman P, Darfeuille-Michaud A and Barnich $\mathrm{N}$ : Diet-induced hypoxia responsive element demethylation increases CEACAM6 expression, favouring Crohn's diseaseassociated Escherichia coli colonisation. Gut 64: 428-437, 2015.

27 Collins D, Hogan AM, and Winter DC: Microbial and viral pathogens in colorectal cancer. Lancet Oncol 12: 504-512, 2011.

28 Tlaskalová-Hogenová H, Stepánková R, Hudcovic T, Tucková L, Cukrowska B, Lodinová-Zádníková R, Kozáková H, Rossmann P, Bártová J, Sokol D, Funda DP, Borovská D, Reháková Z, Sinkora J, Hofman J, Drastich P and Kokesová A: Commensal bacteria (normal microflora), mucosal immunity and chronic inflammatory and autoimmune diseases. Immunol Lett 93: 97-108, 2004. 
29 Kostic A D, Xavier RJ, and Gevers D: The microbiome in inflammatory bowel disease: current status and the future ahead. Gastroenterology 146: 1489-1499, 2014.

30 Wang W, Jovel J, Halloran B, Wine E, Patterson J, Ford G, O'Keefe S, Meng B, Song D, Zhang Y, Tian Z, Wasilenko ST, Rahbari M, Reza S, Mitchell T, Jordan T, Carpenter E, Madsen K, Fedorak R, Dielemann LA, Ka-Shu Wong G and Mason AL: Metagenomic analysis of microbiome in colon tissue from subjects with inflammatory bowel diseases reveals interplay of viruses and bacteria. Inflamm Bowel Dis 21: 1419-1424, 2015.

31 Marchesi JR, Adams DH, Fava F, Hermes GD, Hirschfield GM, Hold G, Quraishi MN, Kinross J, Smidt H, Tuohy KM, Thomas LV, Zoetendal EG and Hart A: The gut microbiota and host health: A new clinical frontier. Gut 65: 330-339, 2016.

32 Elliott TR, Rayment NB, Hudspith BN, Hands RE, Taylor K, Parkes GC, Prescott NJ, Petrovska L, Hermon-Taylor J Brostoff J, Boussioutas A, Mathew CG, Bustin SA and Sanderson JD: Lamina propria macrophage phenotypes in relation to Escherichia coli in Crohn's disease. BMC Gastroenterol 15: 75-79, 2015.

33 Fischer H, Holst E, Karlsson F, Benoni C, Toth E, Olesen M, Lindén $\mathrm{M}$ and Sjöberg K: Altered microbiota in microscopic colitis. Gut 64: 1185-1186, 2015.

34 Gevers D, Kugathasan S, Denson LA, Vázquez-Baeza Y, Van Treuren W, Ren B, Schwager E, Knights D, Song SJ, Yassour M, Morgan XC, Kostic AD, Luo C, González A, McDonald D, Haberman Y, Walters T, Baker S, Rosh J, StephensM, Heyman M, Markowitz J, Baldassano R, Griffiths A, Sylvester F, Mack D, Kim S, Crandall W, Hyams J, Huttenhower C, Knight R and Xavier RJ: The treatment-naive microbiome in new-onset Crohn's disease. Cell Host Microbe 15: 382-392, 2014.

35 Yang Y, Wang X and Huycke T: Colon macrophages polarized by commensal bacteria cause colitis and cancer through the bystander effect. Transl Oncol 6: 596-606, 2013.

36 Armstrong H, Bording-Jorgensen M, Dijk S and Wine E: Complex interplay between chronic inflammation, the microbiome, and cancer: Understanding disease progression and what we can do to prevent it. Cancers 10: 83-86, 2018.

37 Foersch S and Neurath MF: Colitis-associated neoplasia: Molecular basis and clinical translation. Cell Mol Life Sci 71: 3523-3535, 2014.

38 Ong HS and Yim HCH: Microbial factors in inflammatory diseases and cancers. Adv Exp Med Biol 1024: 153-174, 2017.

39 Coleman OI and Haller D: Bacterial Signaling at the intestinal epithelial interface in inflammation and Cancer. Front Immunol 8: 1-10, 2018

$40 \mathrm{Hu}$ Y, Leu R, Christophersen CT, Somashekar R, Conlon M, Meng X, Winter JM, Woodman RJ, McKinnon R and Young GP: Manipulation of the gut microbiota using resistant starch is associated with protection against colitis-associated colorectal cancer in rats. Carcinogenesis 37: 366-375, 2016.
41 Fuller AK, Bice BD, Venancio AR, Crowley OM, Staab AM, Georges SJ, Hidalgo JR, Warncke AV and Angus-Hill ML: A method to define the effects of environmental enrichment on colon microbiome biodiversity in a mouse colon tumor model. J Vis Exp 132, 2018. doi: 10.3791/57182

42 Tlaskalova-Hogenova H, Vannucci L, Klimesova K, Stepankova R, Krizan J, and Kverka M: Microbiome and colorectal carcinoma: insights from germ-free and conventional animal models. Cancer J 20: 217-224, 2014.

$43 \mathrm{Wu}$ S, Rhee K, Albesiano E, Rabizadeh S, Wu X, Yen H, Huso DL, Brancati FL, Wick E and McAllister F: A human colonic commensal promotes colon tumorigenesis via activation of Thelper type 17 T-cell responses. Nat Med 15: 1016-1022, 2009.

44 Liang Q, Chiu, J, Chen Y, Huang Y, Higashimori A, Fang J, Brim H, Ashktorab H, Ng SC and Ng SS: Fecal bacteria act as novel biomarkers for noninvasive diagnosis of colorectal cancer. Clin Cancer Res 23: 2061-2070, 2017.

45 O'Keefe SJ: Diet, microorganisms and their metabolites, and colon cancer. Nat Rev Gastroenterol Hepatol 13: 691-706, 2016.

46 Pascal V, Pozuelo M, Borruel N, Casellas F, Campos D, Santiago A, Martinez X, Varela E, Sarrabayrouse G and Machiels K: A microbial signature for Crohn's disease. Gut 66: 813-822, 2017.

47 Purchiaroni F, Tortora A, Gabrielli M, Bertucci F, Gigante G, Ianiro G, Ojetti V, Scarpellini E and Gasbarrini A: The role of intestinal microbiota and the immune system Eur Rev Med Pharmacol Sci 17: 323-333, 2013.

48 Luddy KA, Robertson-Tessi M, Tafreshi NK, Soliman H, and Morse DL: The role of toll-like receptors in colorectal cancer progression: evidence for epithelial to leucocytic transition. Front Immunol 5: 429-435, 2014.

49 Zmora N, Levy M, Pevsner-Fishcer $M$ and Elinav E: Inflammasomes and intestinal inflammation. Mucosal Immunol 10: 865-883, 2017.

50 Chen GY, Shaw MH, Redondo G and Nunez G: The innate immune receptor NOD1 protects the intestine from inflammationinduced tumorigenesis. Cancer Res 68: 10060-10067, 2008.
Received May 1, 2018

Revised May 22, 2018

Accepted May 30, 2018 